\title{
Nachruf auf Prof. Dr. med. G. Siebert
}

Prof. Dr. med. Günther Siebert ist an der Schwelle zum 72. Lebensjahr durch einen raschen Tod von schwerer Krankheit erlöst worden.

Prof. Siebert begann seine Laufbahn nach Kriegsende am Physiologisch-chemischen Institut der Universität Mainz bei Konrad Lang und wurde dort 1951 habilitiert. In diesr Zeit leistete er Pionierarbeit bei der Isolierung, Reindarstellung und Charakterisierung subzellulärer Fraktionen und der Erforschung der Enzymausstattung und des Stoffwechsels von Zellkernen. Unterbrochen durch einen einjährigen Forschungsaufenthalt in New York bei Severo Ochoa mit der Reindarstellung und Charakterisierung der Isocitratdehydrogenase, lag der Schwerpunkt seiner Arbeiten in Mainz bei enzymologischen und biochemischen Fragen.

1967 folgte Siebert einem Ruf auf den Lehrstuhl für Biochemie und Ernährungswissenschaft an der Universität Stuttgart-Hohenheim, deren Rektor er in einem sehr turbulenten akademischen Jahr war. In Hohenheim befaßte er sich mit Biochemie der Ernährung, und hier begann sein Interesse am Stoffwechsel von Zuckeraustauschstoffen.

1979 nahm er einen Ruf auf den Lehrstuhl für experimentelle Zahnheilkunde an der Universität Würzburg als Nachfolger von F. Bramstedt an. Dort brachte er mit bahnbrechenden Untersuchungen zum Stoffwechsel, zur Resorption und zur energetischen Verwertung verschiedener Zuckeraustauschstoffe, die ihm internationalen Ruf eingetragen haben, frischen Wind in die biochemisch-experimentell nicht allzu aktive deutsche Zahnheillkunde.

Zahlreiche Publikationen in internationalen Zeitschriften stammen aus seiner Feder. Eines der letzten Werke, das „stufenweise Prüfschema für Zuckeraustauschstoffe", wird richtungweisend bleiben. Von hohem Niveau und international besucht waren die Würzburger Zuckersymposien.

Siebert war von 1970 bis 1972 Vizepräsident und von 1972 bis 1974 Präsident der Deutschen Gesellschaft für Ernährung. Der Ernährungsbericht 1976 entstand unter seiner Redaktion, und auch in den folgenden Jahren übernahm er immer wieder Einzelkapitel, so noch im Bericht 1988 den Abschnitt „Zuckeraustauschstoffe und Süßstoffe".

Der Zeitschrift für Ernährungswissenschaft stand er nicht nur als Autor, sondern viele Jahre auch im wissenschaftlichen Beirat zur Verfügung.

Die biochemisch orientierte Ernährungswissenschaft hat mit Siebert einen originellen Wissenschaftler voller Ideen und Anregungen verloren, der mit großer Tatkraft so manches Projekt in Bewegung gesetzt hat und dessen scharfsinnige Kritik manchmal gefürchtet, aber immer beachtenswert war.

K. H. Bässler (Mainz) 